\title{
СОГЛАСУЮЩИЙ НЕЧЕТВЕРТЬВОЛНОВЫЙ ТРАНСФОРМАТОР
}

\author{
БЕЛЯВЦЕВ В. Б., ПРИЙМАК В. Ю., МАРКУС А. Т.
}

Харьковский наииональный университет радиоэлектроники, Украина, Харьков, 61166, пр-т Ленина, 14

\begin{abstract}
Аннотация. Предложен нечетвертьволновый трансформатор для узкополосного согласования нагрузки с полным сопротивлением. Отсутствие отражения достигается выбором соответствующих друг другу размеров поперечного сечения согласующей секции и ее длины
\end{abstract}

Ключевые слова: узкополосное согласование; трансформатор; волновое сопротивление; входное сопротивление; полное сопротивление; коэффициент стоячей волны; КСВ

\section{ПОСТАНОВКА ЗАДАЧИ}

Один из вариантов узкополосного согласования нагрузки с полным сопротивлением основан на применении так называемого четвертьволнового трансформатора [1-3]. Между подводящей линией с волновым сопротивлением $Z_{\text {в } 1}$ (рис. 1) и рассогласованной линией с волновым сопротивлением $Z_{\text {в3 }}$, нагруженной на полное сопротивление $\underline{Z}_{\mathrm{H}}$, располагают согласующую секцию длиной $l_{2}=\Lambda_{2} / 4$, где $\Lambda_{2}$ - длина волны в отрезке линии с волновым сопротивлением $Z_{\mathrm{B} 2}=\sqrt{Z_{\mathrm{B} 1} Z_{\mathrm{B} 3} K}$.

При этом выбором длины $l_{3}$ обеспечивают на входе третьей линии, т.е. на выходе четвертьволновой секции, входное сопротивление чисто активным, что соответствует или максимуму стоячей волны на этом входе ( $K=$ КСВ), или минимуму ( $K=1 / \mathrm{KCB})$, где КСВ коэффициент стоячей волны.

В отличие от варианта четвертьволнового трансформатора поставлена задача определения характеристик согласующего трансформатора с произвольной длиной $l_{2} \neq \Lambda_{2} / 4$ и на- грузкой, подключенной непосредственно к выходу согласующей секции $\left(l_{3}=0\right)$.

\section{РАСЧЕТНЫЕ ФОРМУЛЫ}

Полное входное сопротивление третьей линии является нагрузкой второй секции, поэтому

$$
\underline{Z}_{\mathrm{B} \times 3}=R_{\mathrm{B \times} 3}+\mathrm{i} X_{\mathrm{B \times} 3}=\underline{Z}_{\mathrm{H} 2}=R_{\mathrm{H} 2}+\mathrm{i} X_{\mathrm{H} 2} .
$$

С учетом входного сопротивления второй секции $\underline{Z}_{\text {вх2 }}$, волнового сопротивления подводящей линии $Z_{\text {в1 }}$ и коэффициента фазы $\beta_{2}=2 \pi / \Lambda_{2}$ комплексный коэффициент отражения на стыке первых двух линий запишется в виде

$$
\begin{gathered}
\underline{\rho}_{2}=\frac{\underline{Z}_{\mathrm{B} 2}-Z_{\mathrm{B} 1}}{\underline{Z}_{\mathrm{B} \times 2}+Z_{\mathrm{B} 1}}, \\
\underline{Z}_{\mathrm{B} 2}=Z_{\mathrm{B} 2} \frac{\underline{Z}_{\mathrm{H} 2}+\mathrm{i} Z_{\mathrm{B} 2} \operatorname{tg}\left(\beta_{2} l_{2}\right)}{Z_{\mathrm{B} 2}+\mathrm{i} \underline{Z}_{\mathrm{H} 2} \operatorname{tg}\left(\beta_{2} l_{2}\right)}= \\
=Z_{\mathrm{B} 2} \frac{R_{\mathrm{B} \times 3}+\mathrm{i}\left(X_{\mathrm{B} \times 3}+Z_{\mathrm{B} 2} \operatorname{tg}\left(\beta_{2} l_{2}\right)\right)}{\left(Z_{\mathrm{B} 2}-X_{\mathrm{B} 3} \operatorname{tg}\left(\beta_{2} l_{2}\right)\right)+\mathrm{i} R_{\mathrm{B} \times 3} \operatorname{tg}\left(\beta_{2} l_{2}\right)} .
\end{gathered}
$$

